\title{
Cladding-pumped Raman fiber amplifier
}

\author{
J. Nilsson, J. K. Sahu, J. N. Jang, R. Selvas, D. C. Hanna \\ Optoelectronics Research Centre, University of Southomptom, Southompton SOI7 IBJ, Englond \\ phone: +44 238059 3101, fax: +44238059 3142, email:jn@orc.soton.ac.uk \\ A. B. Grudinin \\ Southampton Photonics, Phi House, Enterprise Road, Chilworth Science Park, Southampton SO16 NNS, England
}

Introduction: Cladding-pumped rare-earth-doped fibers and Raman fiber devices have been two of the most compelling advances in the area of high-power fiber amplifiers and lasers in recent years. Cladding-pumping enables pumping with multi-mode pump sources that deliver high-power at a low cost. The core of the double-clad fiber can still be single-moded, so that single-mode amplification or lasing can be realized. Raman gain requires high pump. powers and Raman conversion is therefore only efficient at high power levels. On the other hand, Raman devices are very flexible as gain is available at arbitrary wavelengths. with the right pump source. Cladding-pumped Raman fiber devices represent a natural progression to multi-mode pumping and Raman fiber devices [1]. While cladding-pumped Raman fiber devices require pump powers of, say, $100 \mathrm{~W}$, such powers are available from fiber sources as well as from traditional bulk sources. For example, a multimode Nd-doped fiber laser with $1 \mathrm{~kW}$ of output power was recently reported [2]. With pulsed sources, however, the required peak powers can be achieved with relatively modest average powers. Here, we report for the first time Raman amplification in a cladding-pumped fiber, without any rare-earth doping. We believe that this is an important step towards a whole class of new optical amplifier devices based on brightness-enhancing nonlinear conversion of multimode pumps, including not only Raman converters but also Brillouin and optical parametric converters. $[3,4]$

Set-up: Our experimental set-up is shown in Fig. 1. We used a Q-switched $\mathrm{Er}$ - $\mathrm{Yb}$ co-doped fiber laser in a multi-fiber (GT-wave) arrangement. Here, we used the laser $8.7 \mathrm{kHz}$ repetition rate, where it generated pulses at $1565-$ $1570 \mathrm{~nm}$ with energies of $30 \mu \mathrm{J}$ and durations of $210 \mathrm{~ns}$. Thus, the peak power became $140 \mathrm{~W}$, or 540 times the average power. The time jitter was $\sim 5$ ns. The output from the fiber laser was free-space coupled into the double-clad Raman fiber (DCRF) via a dichroic mirror. Though the Q-switched fiber laser produced a single-moded output, we took great care to ensure that the pump beam was launched into the inner cladding, rather than the core, of the DCRF. Following all measurements presented here, we cut the DCRF a small distance from the pump launch end, and evaluated first total launched pump power and then power in the core by splicing the DCRF to a piece of standard single-mode fiber. We could launch up to $88 \%$ of the output power from the Q-switched fiber laser into DCRF, $10-$ $15 \%$ of which was in the core. We varied the launched pump power by inducing a bend-loss on the output fiber of the Q-switched fiber laser, without changing the pulse shape, repetition rate, relation between peak and average power, or fraction of power in the core of the DCRF.

In the DCRF, the pump source generates Raman gain with a peak wavelength of $1680-1690 \mathrm{~mm}$. We did not have a signal seed source at that wavelength, and therefore had to resort to the rather complicated set-up of Fig. 1, which effectively generates its own signal seed. However, the Raman converter itself is very simple, consisting only of the DCRF. The pump-to-signal conversion takes place in a single amplification pass. Thus, we consider the device under test to be the cladding-pumped Raman fiber, with signal and pump beams launched into its left end in Fig. 1. Alternatively, the set-up can be viewed as a synchronously pumped pulsed Raman laser.

The DCRF had a pure silica outer cladding and germanosilicate inner cladding and core. The inner cladding had a diameter of $21.6 \mu \mathrm{m}$ and an NA of 0.22 with respect to the outer cladding. The core had a diameter of $9 \mu \mathrm{m}$ and an NA of 0.14 with respect to the inner cladding (estimated cut-off wavelength $1630 \mathrm{~nm}$ ). The core loss was $3.1 \mathrm{~dB} / \mathrm{km}$ and the inner cladding was $2.3 \mathrm{~dB} / \mathrm{km}$, both at $1550 \mathrm{~nm}$. We used different fiber lengths of around $1 \mathrm{~km}$. Since Raman gain is essentially instantaneous and since the pump pulse is much shorter than the DCRF, the pump pulse creates Raman gain that travels with it through the fiber. This makes the Raman gain essentially uni-directional, and the signal at the output end of the DCRF will also be pulsed, temporally coincident with the pump pulse.

At its output end, the DCRF is spliced to a fused fiber coupler fabricated with standard single-mode fiber (NA 0.12 , core diameter $8 \mu \mathrm{m}$ ). The splice loss between standard single-mode fiber (SSMF) and the DCRF is $\sim 0.5 \mathrm{~dB}$ for the signal traveling in the core mode. By contrast, since the cladding in the SSMF does not guide light, essentially all pump light is lost here. The coupler had a nearly flat wavelength response, and coupled out $25 \%$ of the incident power at $\sim 1680 \mathrm{~nm}$. This monitor coupler was then spliced to another, wavelength-selective, coupler. It had a low 
transmission loss $(\sim 2 \mathrm{~dB})$ at the first stokes wavelength $(-1680 \mathrm{~nm})$ but a high loss at the second stokes wavelength as well as at the pump wavelength. Thus, it suppressed higher-order stokes generation. The couplers were followed $1690 \mathrm{~nm} 5 \mathrm{~dB}$ ). A high-reflectingt allowed us to change the cavity loss. Finally, there was $\sim 10 \mathrm{~km}$ of SSMF (loss at $2 \mathrm{~dB}$. The long SSMF delayed the signal feed butted to the fiber in the far end with an estimated reflection loss of The signal pulse is reflected

reflected again from the perpend through the cavity all the way to the pump launch end of the DCRF. There, $4 \%$ is DCRF. The reflected signal light acts as a seed fiber end. At the same time, a new pump pulse is launched into the Because the mode-selection that occurs in the fSM conversion in the cladding-pumped Raman fiber amplifier. reflected signal is almost exclusively coupled to time was $115.27 \mu \mathrm{s}$. The Q-switched fiber the core mode. The roundtrip cavity loss was $\sim 55$ dB. The roundtrip the effective length of the DCRF without changing carefully adjusted to this for synchronous pumping. We varied Fig. 1 to a position between the VOA and the $10 \mathrm{~km} \mathrm{SSMF,} \mathrm{where} \mathrm{the} \mathrm{DCRF} \mathrm{is} \mathrm{un-pumped.}$

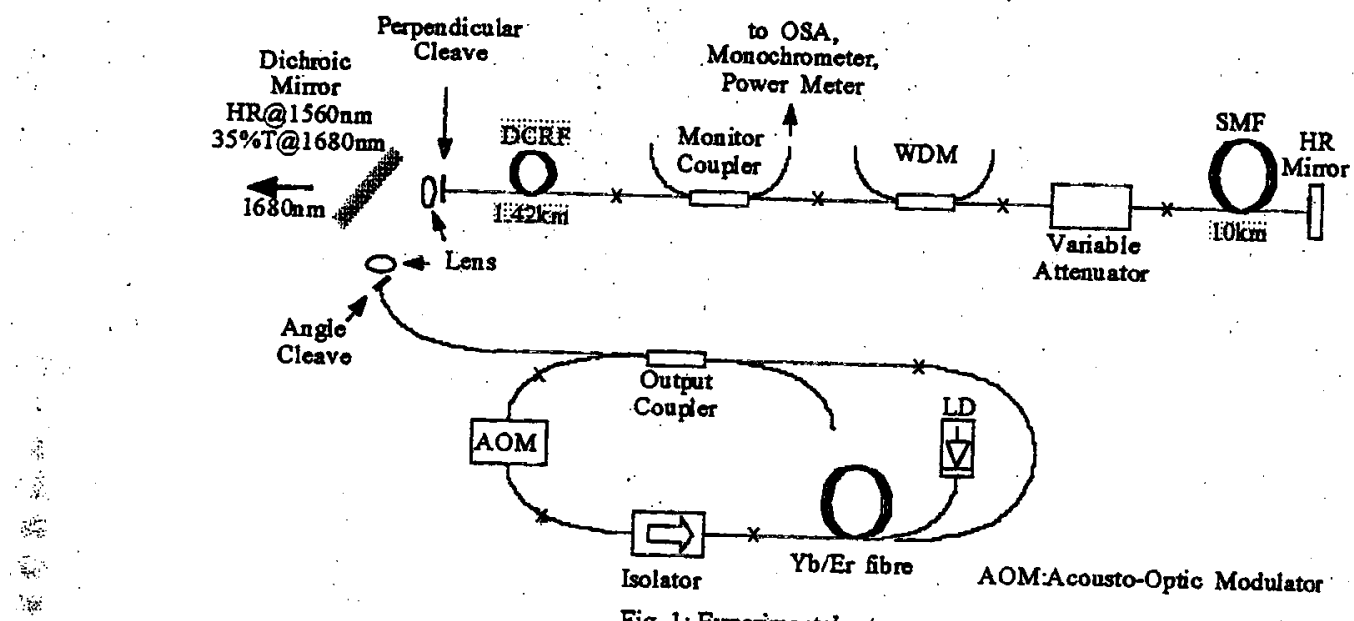

Fig. 1: Experimental set-up.

Results: Figure 2 shows the DCRF gain vs. pump power for 1420 and $940 \mathrm{~m}$ long DCRFs. The gain was determined by varying the total cavity loss via the VOA, and adjusting the pump power until threshold for lasing was reached. The gain slopes are $1.5 \mathrm{~dB} / \mathrm{mW}$ and $1.0 \mathrm{~dB} / \mathrm{mW}$, respectively, or $2.8 \mathrm{~dB} / \mathrm{W}$ and $1.9 \mathrm{~dB} / \mathrm{W}$ with respect to peak power. The effective lengths become $1000 \mathrm{~m}$ and $740 \mathrm{~m}$. These numbers are in good agreement with theory, given the uncertainty in evolution of polarization and modal power distribution, and that the high Ge-content increases the
Raman cross-section.

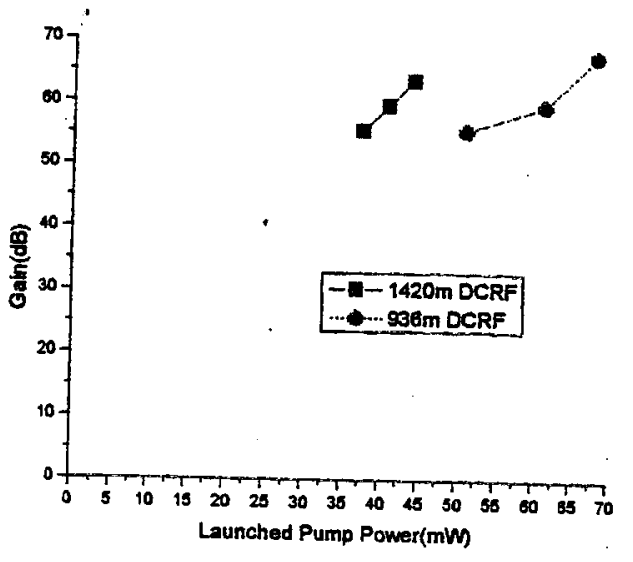

Fig. 2: Gain vs. pump power.

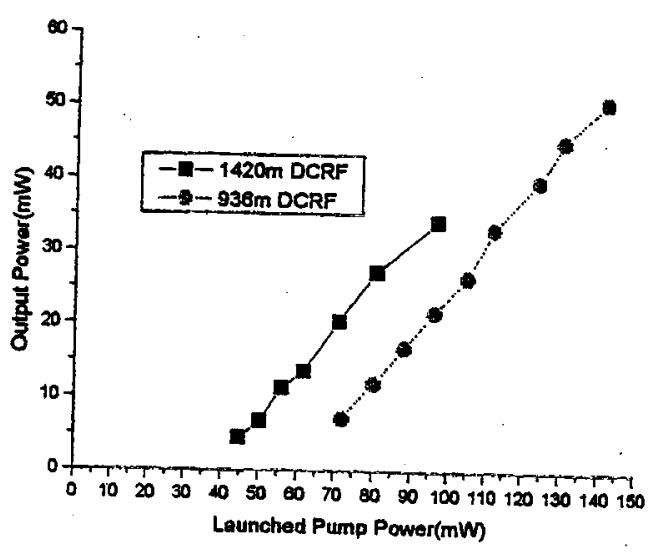

Fig. 3: Output power vs. pump power.

Figure 3 shows the output power from the DCRF vs. pump power for 1420 and $940 \mathrm{~m}$ long DCRFs, with the VOA at its minimum loss value. The output power was evaluated by measuring the power exiting the coupler monitor port 
and recalculating it to the power coming out from the DCRF. The thresholds are $37 \mathrm{~mW}$ and $61 \mathrm{~mW}$, and the slope efficiencies are $60 \%$ and $64 \%$ for the longer and shorter fiber, respectively. For both fiber lengths, the power in the core at the output end becomes significantly higher than in the input end: The highest pump power launched into the core was $10-20 \mathrm{~mW}$. This demonstrates brightness enhancement via Raman amplification of a signal in the core with a pump beam substantially launched into the inner cladding.

At high pump power, higher-order Raman generation can occur. In Fig 3, we restricted the pump power to values for which this was negligible. Thus, since higher-order generation occurs more readily in longer fibers, the maximum pump power (and ouptut power) is lower for $1420 \mathrm{~m}$ than for $940 \mathrm{~m}$ fiber length. Figure 4 shows the output spectrum for a fiber length of $940 \mathrm{~m}$ and a pump power of $140 \mathrm{~mW}$. Unfortunately, the spectrum analyzer was limited to wavelengths up to $1750 \mathrm{~nm}$, whereas the second stokes occurred at $\sim 1810 \mathrm{~nm}$. However, we also used a monochromator to resolve higher-order stokes radiation and for temporal measurements. Figure 5 shows output pulse shapes at the wavelengths of the pump and first and second stokes beams, measured with the monochromator and a germanium detector. The second stokes signal is much weaker than the first stokes signal.

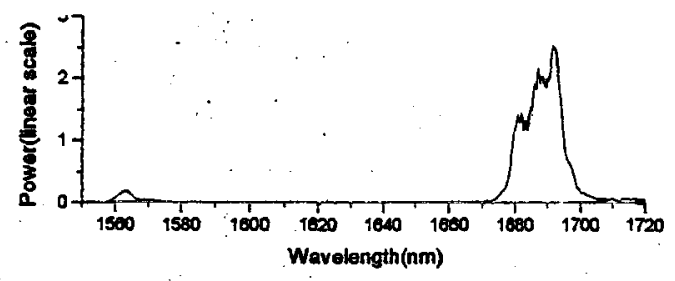

Fig. 4:-Output spectrum after $940 \mathrm{~m}$ of DCRF.

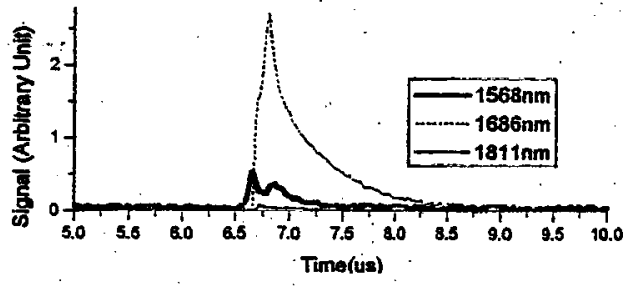

Fig. 5: Output pulse shapes

Discussion: Despite the low Raman gain efficiency, it is relatively straightforward to reach high gain with pulsed pump sources. Even $\mathrm{cw}$ pump lasers (fiber and non-fiber ones) of sufficiently high powers are becoming increasingly available. In a laser configuration, the cavity losses can be sufficiently low for thresholds of a few watts, given a gain efficiency of the order of $1 \mathrm{~dB} / \mathrm{W}$. Still, cladding-pumped Raman fibers require inner-cladding diameters of a few tens of microns rather than hundreds of microns as is common with rare-earth doped cladding-pumped fibers. This limits the scope for brightness enhancement and the high pump intensities required presently preclude direct diode pumping. On the other hand, there are several advantages of Raman devices over rare-earth ones. Besides the flexibility in wavlength, the low loss of germanosilicate fibers allows the use of much longer devices, so that the heating per unit length can be small. The small quantum defect of Raman scattering in silica further simplifies thermal management.

The length of the Raman fiber converter is important. A longer fiber leads to a higher gain efficiency in the smallsignal regime, making it easier to reach threshold with a longer fiber. However, for higher powers, secondary Raman scattering occurs, this too with lower threshold for longer fibers. To avoid this, the pump power used with a given fiber length must be limited. A shorter fiber has a higher limit. In Fig. 3, for pump powers up to $100 \mathrm{~mW}$, the $1420 \mathrm{~m}$ fiber is better than the $940 \mathrm{~m}$ one. For higher pump powers, second-order Raman scattering occurs in the $1420 \mathrm{~m}$ fiber. One can then reach higher output powers with $940 \mathrm{~m}$ of fiber than with $1420 \mathrm{~m}$, with appropriate pump power. (Though a wavelength-suppressing filter could be used to suppress higher-order Raman scattering, this may be difficult in practice.) Raman scattering in the cladding should also be avoided. Since the core and cladding compositions are quite similar, and since the pump intensity is essentially similar in the core and inner cladding, the Raman gain will be similar in the core and inner cladding. If a single-moded output is required, one must then seed the core-mode (or provide mode-selective feedback in a laser)

Conclusions: In conclusion, we report for the first time results on amplification in a cladding-pumped Raman fiber. We used a Q-witched Er-Yb co-doped fiber laser operating at $1565-1570 \mathrm{~nm}$ as a pump source. The power conversion efficiency was up to $36 \%$, with a slope of $64 \%$. We believe that cladding-pumped fiber devices present a very exciting alternative for amplification and brightness conversion at high powers.

1. R. R. Rice, "Multimode Raman fiber amplifier and method", US patent no. 6,353,087 (2002)

2. K.-I. Ueda, H. Sekiguchi, and H. Kan, “1 kW cw output from fiber embedded lasers”, in Proc. Conference on Lasers and Eloctro-Optics, Long Beach, USA, 2002, post-ieadline paper CPDC4

3. B. C. Rodgers, T. H. Russell, and W. B. Rob, "Laser beam combining and cleanup by stimulated Brillouin scattering in a multimode optical fiber", Opt Lett. 24, 1124-1126 (1999)

4. A Piskarskas, V Smilgevicius, A Stabinis, “Optical parametric oscillation excited by an incoherent conical beam”, Opt Comm 143, 72-74 (1997) 